\title{
Review
}

\section{Movement and the ordering of freedom: On liberal governances of mobility}

\author{
Hagar Kotef \\ Duke University Press, Durham and London, 2015, xiii+234 pp., \\ ISBN: 978-0822358435
}

Contemporary Political Theory (2016) 15, e29-e32. doi:10.1057/cpt.2015.44;

published online 28 July 2015

Hagar Kotef's enquiry into 'the politics of motion' is timely, excellently written and surely a must read for researchers not just of surveillance/control societies and of Israel-Palestine (the book's regional focus), but more broadly for scholars in cultural politics. Yet, this is not a book only for professionals. An enquiry into the politics of motion - as Kotef explains in the introduction - is an enquiry into forms of citizenship and non-citizenship and therefore, although not all sections are fully accessible, the book is stimulating reading for broader audiences attempting to comprehend how fetters on movement have to do with the kind of persons we are. In this sense Movement and the Ordering of Freedom is an important contribution to the growing interest in political subjectivity. Rather than keep spiralling around narratives of oppression that help very little to understand how to deterritorialise our oppressive existential territories, Kotef's cultural politics diverts from these conservative trends in the radical writing about Israel-Palestine. Kotef's book also has a unique value, which is to task the rescuing of traditional political theory to explain aspects of our present predicament. This appreciation has little to do with nostalgia. Wrongly, too many post-structuralist scholars in the social sciences and the humanities find no reason to maintain the philosophical dialogue with the past as one more vivid source of thought. Kotef's therefore provides a welcome contribution to contemporary political theory.

The main argument of the book is that by looking into the liberal tradition in political theory we might be able to explain the relation between the kind of beings we are and the kind of regimes of movement that characterise our potentialities these two systems are two interrelated faces of the same social formation. As Kotef states, 'Regimes of movement are thus never simply a way to control, to regulate, or to incite movement; regimes of movement are integral to the formation of different modes of being' (p. 15). Kotef unfolds and demonstrates this main argument by discussing four propositions (see summary on pp. 3-6): (i) subject-positions and the 
political order within which they gain meaning cannot be divorced from movement; (ii) the 'capacity of locomotion' has a historical role in the construction of the privileged subject-position of the liberal subject; (iii) liberal mobility was cultivated as a regulated historical construction, particularly, for the movement of liberal subjects to thrive, subaltern subjects' movement had to be managed; and (iv) the historical split in the configuration of movement (previous argument) has contemporary spatial implications that Kotef amply illustrates as this takes place in the Israeli regime of occupation in the Palestinian territories.

These four propositions however, are not discussed in the book in a traditional linear fashion, first 'theory' then 'practice'. This makes the reading engaging. Kotef's line of work clearly goes beyond the all too empirical portrays of Israel's power (which at times seem to expose an aversion to 'the concept') overflowing the literature on the topic these days, and it also offers an alternative to the more deductive writing that makes the evaluation of the actual no more than a reflection of theory. Thus, following the introduction, the first two chapters rapidly submerge the reader into the story of Israel's technological regime of movement in the West Bank and Gaza that was consolidated during the period between the 1993 Oslo Accords and 'the years following the El Aqsa Intifada' after year 2000 (p. 28). The chapter 'Between Imaginary Lines' (pp. 27-51) explains how the making of the Palestinians 'unruly or undisciplinable subjects' - by way of creating conditions that structurally impede self-governance - helps Israel to justify the continuation of the occupation and to indefinitely defer engaging in a sincere peace process. The check point, illustrates Kotef, is one such artefact of subjectification; basically, it becomes so by constructing a 'failure' (implemented in the adoption of unclear procedures, constant change of rules, making thresholds imperceptible, the use of Hebrew and so forth) that Palestinians cannot outdo. In other words, Palestinians are made into active transgressors by always displacing at Israel's will the possibility of obeying. What this chapter makes abundantly clear is that subjectification is the process by means of which power establishes itself, not formal sovereignty. Kotef's contribution is centred on how the structuration of movement is part and parcel of processes of subjectification. The subsequent chapter, 'A Tale of Two Roads', adds to this understanding of the role of movement in subjectification by looking into how the separation of movement between Jewish-Israelis and Palestinians - in roads epitomises the fundamental Zionist maxim: 'You should not mix'. As Kotef implies, when it comes to the potentiality of sharing living spaces, roads included, the Israeli regime is unambiguous (p. 56).

It is only in the third chapter that Kotef unfurls a genealogy of motion and freedom from the liberal tradition that eventually explains why today, in the global discursive arena, Israel is able to 'make sense' of its rule of the Palestinians by invoking the failure of Palestinians to self-regulate their movement - namely, their failure to become liberal subjects. Kotef looks into the works of Hobbes, Kant, Wollstonecraft and Locke to ultimately suggest that, in the liberal tradition, motion arises as the 
matter of freedom alongside 'limits of many kinds' (p. 86), limits that are fundamentally expressed as self-regulatory, a tradition that anyone who has spent some years in a western society can confirm - that rational self-regulatory attitude or the irritating white demand for 'moderation'. The impacts of the liberal equation of subjectivity - freedom becomes enjoyable through inner (consented) rather than external impediments on the virtuality and actuality of motion ${ }^{1}-$ are further explored in Chapter 4 where Kotef shifts the focus 'to those who presumably lacked such mechanisms of restraint, whose movement was deemed as excessive' (p. 88). Here the reader will find a theme that stays also in the following chapters: the subjectifying relation between those bodies judged as natural transgressors, the dividing modes of governance that this transgression entails (Kotef focuses on the racial/geographical division and on the division forced by property and land) and the discourses that justify the divisions and their accompanying hierarchies. From Plato, Kotef emphasises the contrast of the desired stability that the few can attain to the excessive movement of the demos (pp. 90-93); from Hobbes, the question of movement as a problem of violence and security (pp. 93-100), and from Locke, the radicalisation of the split between safe/free and unsafe/unfree movement (pp. 101-111). These negotiations lead indeed to a corollary: from the standpoint of power, colonialism and particularly settler colonialism became defensible on grounds of the anthropological liberal series: rationality-organisation-division-enclosure-stability-selfregulation. Any subjectivity below or beyond would be deemed as excess.

Kotef's fifth chapter (pp. 112-135) puts a research agenda in place. By asserting that movement can be thought of as the principle of collective bodies, it deals with the question of what are 'the political implications of a political paradigm pivoting around the movement of colletivities?' (p. 113). It launches an introductory exploration of this question mainly in regard to the state (consolidation versus expansion) and the colony, but also of people, social movements and resistance. A transition takes the reader into the last chapter where Kotef discuss justification the mechanism that distinguishes between freedom and violence as the two liberal modes of movement.

Particularly after WWII it has become difficult to justify discrimination against groups just because of their identities. I find hard it to claim that humanity did internalise the significance or the sentiment involved in the idea of human rights; rather, it is the public relations of human rights in the international arena that makes it difficult to justify violence against the subaltern as such, and as Kotef puts it, it is as if states cannot bear their own violence for too long unless it is anchored in some ethical schema' (p. 138). Kotef's paradigm - 'schemas of identity are formed in tandem with schemas of mobility' (p. 138) - helps to explain how certain identities become punishable as 'Patterns of mobility have functioned, and still do, to convert these identities into punishable ... practices' (p. 139). This is about thinking violence through the historical constraints of mobility. So for instance, if the Palestinian citizens of Israel find almost impossible to buy or rent a house in a non-Arab 
community, town or city in Israel, it is not because they are Palestinians - "we are not racist', many Israelis would say - but because they fall short of becoming part of Israel's public canon and therefore they are unfit to share housing spaces, regardless of the fact that this lack of socio-cultural mobility is the way by which Zionism positioned Palestinian subjectivities as an inclusive exclusion.

As said, Kotef's is a book on subjectivity, and as such, it forces us to think through the historical coordinates of Israeli and Palestinian subjectivities, shaped and reshaped in the settler colonial encounter. The point of such introspection lies in its contribution to how to do away with settler colonial life, or it does not have a point at all. In that respect, I also take from Kotef's book the message it conveys for JewishIsraelis, a political yet strongly personal message: as an exercise not on selfconstraint, personal and collective social transformation kicks off with the erasure of the 'imaginary lines' that a subaltern should not ever cross - a much needed change in the constitution of settler-colonial subjectivity.

\section{Note}

1 I'm using the notions of 'virtuality' and 'actuality' in the Deleuzian sense, as the two complementary and always interconnected dimensions of the real.

Marcelo Svirsky

University of Wollongong, Wollongong 2522 NSW, Australia msvirsky@uow.edu.au 